\title{
Microbiological Hygiene Quality of Thermal Muds: A Pilot Study in Pelotherapy Facilities of the Euganean Thermal District (NE Italy)
}

\author{
Tatjana Baldovin ${ }^{1}$, Irene Amoruso ${ }^{1, * \mathbb{C}}$, Fabrizio Caldara ${ }^{2}$, Alessandra Buja ${ }^{1} \mathbb{D}$, \\ Vincenzo Baldo ${ }^{1}{ }^{1}$, Silvia Cocchio ${ }^{1}$ and Chiara Bertoncello ${ }^{1}$ \\ 1 Department of Cardiac, Thoracic, Vascular Sciences and Public Health, Unit of Hygiene and Public Health, \\ University of Padua, Via L. Loredan, 18-35131 Padua, Italy; tatjana.baldovin@unipd.it (T.B.); \\ alessandra.buja@unipd.it (A.B.); vincenzo.baldo@unipd.it (V.B.); silvia.cocchio@unipd.it (S.C.); \\ chiara.bertoncello@unipd.it (C.B.) \\ 2 Pietro d'Abano Center for Thermal Studies, Via Jappelli, 5-35031 Abano Terme (PD), Italy; \\ fabrizio.caldara@centrostuditermali.org \\ * Correspondence: irene.amoruso@unipd.it
}

Received: 4 June 2020; Accepted: 9 July 2020; Published: 13 July 2020

\begin{abstract}
Evaluation of hygienic aspects of thermal mud microbiology is still neglected. This study evaluates the microbiological hygiene quality of thermal muds, providing a comprehensive assessment of the whole mud cultivation chain. Maturing mud, peloid and used mud samples were collected twice in a year from 30 SPAs of the Euganean Thermal District, NE Italy. Samples were processed with an ad hoc laboratory method. The following indicator parameters were assessed: Total Count at 22, 37 and $55^{\circ} \mathrm{C}$; total coliforms; Escherichia coli; enterococci; Staphylococcus aureus; Pseudomonas aeruginosa; sulfite-reducing clostridia; dermatophytes. Statistical significance of differences between the two sampling campaigns and correlation between temperature and indicator parameters were evaluated. One-hundred eighty samples were analyzed. Widespread presence of environmental species was found, as well as hints of possible microorganism transfer from the patient's skin to the mud. Proper setting of thermal water temperature resulted critical, in terms of hygienic quality. Although optimal maturation should be granted (thermal water at $30-42{ }^{\circ} \mathrm{C}$ ), a pasteurization step at $60-65{ }^{\circ} \mathrm{C}$ is strongly recommended to sanitize peloids before pelotherapy. Facilities re-using thermal muds should also implement a regeneration step at $\geq 65^{\circ} \mathrm{C}$. Core evaluation of thermal mud hygienic quality could encompass the following guidelines: absence (i.e., 0 colony forming units (CFU)/g) of E. coli, P. aeruginosa, S. aureus and dermatophytes.
\end{abstract}

Keywords: thermal mud; pelotherapy; peloids; SPA; microbiological quality

\section{Introduction}

Beneficial properties of clay minerals and especially of thermal muds (TMs) are well known and established: their uses in both historical and modern times are thoroughly discussed in a dedicated review [1]. Among the many curative applications, the ancient practice of pelotherapy has been carried out for centuries worldwide [2,3] and it gained popularity also for wellness purposes [4]. Pelotherapy consists in the application of hot TMs $\left(40 \pm 2{ }^{\circ} \mathrm{C}\right)$ in a thick-layer, directly on the skin of the patient that is then covered with an insulating cloth, in order to preserve heat.

Pelotherapy has a stimulatory, antiphlogistic, analgesic action $[1,4,5]$ and it is recommended either as treatment or adjuvant therapy for rheumatic disorders and other musculoskeletal conditions, e.g., osteoarthritis [6-8], fibromyalgia [9,10], rheumathoid arthritis [9], low back pain [11] and traumatic 
injuries [12]. Pelotherapy is suggested also for other conditions, e.g., dermatological disorders [13], neuro- and vasculopathies [14,15] and to improve stress resilience [16].

The overall quality of TMs is determined by several factors, i.e., composition of raw mud, thermal water characteristics and maturation procedure. In fact, TMs gain their therapeutic properties during the maturation process, in which mud is blended with thermal water under specific conditions [12,17]. Complex inorganic and organic changes of the mud matrix lead to the improvement of physico-chemical, rheological and biological properties required for an effective pelotherapy [1,2,12]. Most pelotherapy centers employ raw muds occurring in situ, but where exploitation of natural reserves is strictly regulated [18], TMs are currently regenerated, i.e., after the first application on the patient they are matured and used anew [4]. In the past, some authors discussed the need to establish standard quality criteria for TMs intended to be used in therapy [3,4]. Several studies evaluated chemical, mineralogical, radiological and granulometric properties of raw muds under the perspective of human health safety e.g., [19-22]. The mobility of hazardous chemical elements possibly contained in clay materials was also assessed with peculiar in vitro leaching tests [23,24].

So far, biological investigations mainly addressed the characterization of thermophilic microorganisms (e.g., diatoms and cyanobacteria) involved in the maturation process and release of therapeutically active biogenic compounds [2,3,25-27]. On the contrary, hygienic aspects of TMs microbiology have been contemplated only by a few studies [3,28-32]. However, no exhaustive research on microbiological hygiene quality of TMs has been published so far. Studies evaluating either the effectiveness of mud cultivation in terms of sanitization or the possible transfer of microorganisms from patient to TM are also missing.

The present work aims at investigating the microbiological quality of TMs from the microbiological hygiene perspective. A dedicated laboratory method was implemented and a set of suitable indicator parameters was tested. Especially, microbial safety of every step of the TM cultivation process was evaluated to highlight critical points and peculiar contamination risks.

\section{Materials and Methods}

\subsection{Sampling Sites}

TM samples were collected from 30 facilities of the Euganean thermal basin (NE Italy), each performing pelotherapy either as private spa or in convention with the Italian National Health System. Each facility was surveyed twice in a year, respectively during the two thermal tourism high seasons, i.e., spring sampling (SS) and autumn sampling (AS).

\subsection{Sample Collection}

From each cultivation plant site, 6 different mud samples were collected. In detail, three different kinds of muds were sampled during both SS and AS: maturing mud (M); peloids (P) and used mud undergoing regeneration (R). A steel core-drilling device with a diameter of $6 \mathrm{~cm}$ was used to collect mud samples. An inner piston collects a mud cylinder of $20 \mathrm{~cm}$ in height, thus sampling the mud layer involved by maturation biological processes [33]. The core-drilling device was rinsed with water and thoroughly flamed with a field Bunsen burner before the collection of a different sample. Each mud sample was extruded from the core-drilling device into a sterile Stomacher ${ }^{\circledR}$ bag (Seward GmbH, Worthing, UK). Samples that could not be collected with the core-drilling device (i.e., automated dispensers) were directly poured inside Stomacher ${ }^{\circledR}$ bags. Samples were carried to the laboratory by cooled transport $\left(4^{\circ} \mathrm{C}\right)$ and processed within $10 \mathrm{~h}$ from collection. Temperature was also recorded for each sampling point.

\subsection{Microbiological Methods}

Prior to analysis, the content of each Stomacher ${ }^{\circledR}$ bag was manually kneaded for $30 \mathrm{~s}$ to roughly uniform the sample. A 1:10 dilution was prepared for each sample by suspending $100 \pm 3 \mathrm{~g}$ of mud in $900 \mathrm{~mL}$ of Dulbecco Phosphate Buffer Saline, inside a sterile 2 L Erlenmeyer flask. The flask was 
then placed on an orbital shaker at $300 \mathrm{rpm}$ for $10 \mathrm{~min}$ or until complete suspension was achieved. The flask was further maintained under slow agitation during laboratory testing procedures, in order to avoid sedimentation of the sample and, possibly, lower bacteria recovery.

Selected indicator parameters were: total viable count (TVC), total coliforms, E. coli, enterococci, S. aureus, P. aeruginosa, and sulfite-reducing clostridia and dermatophytes fungi. Table 1 reports the full list of indicator parameters, growth media (Biolife Italiana, Milano, Italy) and incubation conditions. TVC was evaluated by pour plate method at 22,37 and $55^{\circ} \mathrm{C}$ by seeding $1 \mathrm{~mL}$ of sample. For each sample, multiple dilutions were prepared and tested (i.e., 1:10, 1:100 and 1:1000), in order to obtain results within the countable range of 30-300 colony forming units (CFU) per plate. The other parameters were evaluated by membrane filtration technique. Three different volumes (i.e., 1, 5 and $10 \mathrm{~mL}$ ) of the 1:10 diluted sample were filtered on $0.45 \mu \mathrm{m}$ sterile cellulose acetate membranes (Sartorius-Stedim Biotech, Goettingen, Germany), to grant readability of the plate (i.e., 20-200 CFU per plate). Membranes were then transferred on $\varnothing 60 \mathrm{~mm}$ petri dishes containing dedicated agar media.

Table 1. Microbiological quality indicator parameters. Agar media and growth conditions, i.e., incubation time and temperature, are hereby reported. PCA—Plate Count Agar; C-EC-Chromogenic Coliform agar; TBX-Tryptone Bile X-GLUC agar; SPS—Sulphite Polymyxin Sulphadiazine; DTM-Dermatophyte agar. * SPS agar plates were incubated in anaerobic conditions, within an anaerobic jar (AnaeroJar $2.5 \mathrm{~L}$, Oxoid Ltd., Basingstoke, UK).

\begin{tabular}{cccc}
\hline Indicator Parameter & Agar Medium & Incubation Time (hrs) & Growth Temperature $\left({ }^{\circ}\right.$ C) \\
\hline \multirow{2}{*}{ Total viable count (TVC) } & & 72 & 22 \\
\cline { 3 - 4 } & PCA & 24 & 37 \\
\hline Total coliforms & & 24 & 37 \\
\hline Escherichia coli & C-EC & 24 & 44 \\
\hline Enterococci & TBX & 24 & 37 \\
\hline Staphylococcus aureus & Slanetz and Bartley & 48 & 37 \\
\hline Pseudomonas aeruginosa & Baird-parker & 24 & 37 \\
\hline Sulfite-reducing clostridia & Cetrimide & 24 & 37 \\
\hline Dermatophytes & SPS * & 24 & 30 \\
\hline
\end{tabular}

\subsection{Statistical Testing}

Statistical analysis was conducted with software SOFA Statistics v1.5.2 (Paton-Simpson \& Ass. Ltd, Wellingotn, New Zealand). Non-parametric tests were applied. Wilcoxon Signed Rank Test was used to assess differences between SS and AS paired data. Correlations between temperature and TVCs were evaluated with Spearman's R test. The Mann-Whitney U test was run to assess interaction of temperature with indicator parameters other than TVCs, in terms of presence/absence.

\section{Results}

Relevant characteristics of the 30 facilities considered in the study are described in Table 2. Traditional mud cultivation (T) in $4 \times 4 \times 1 \mathrm{~m}$ concrete tanks was implemented by 26 facilities; 2 facilities employed a mechanized plant with $20 \mathrm{~m}^{3}$ cylindrical iron silos (S) and 2 had a hybrid system (H) with maturation in concrete tanks and storage of TMs in silos. Moreover, distribution of TMs from cultivation area to therapy chambers was also performed with different techniques among different facilities. Direct collection (D) of TMs from maturation tanks and transport with buckets was adopted by 11 sites. The bagnomaria hot water bath technique (B) was used by 15 facilities: buckets were filled with TMs and submerged with thermal water in a dedicated tub for at least $24 \mathrm{~h}$ prior to therapy. A qualified operator carried TM buckets to the therapy chambers when required. The remaining four facilities were furnished with an automated dispenser (A) that piped TMs from the maturation plant to the therapy chamber. Temperatures of each sampling site are also summarized in Table 2. 
Table 2. Characteristics of surveyed facilities and temperature of sampling sites. The table reports the mud cultivation technique (T-traditional tanks, S-mechanized silos, $\mathrm{H}$-hybrid system); thermal mud (TM) distribution method (D—direct collection from tank, B-bagnomaria bucket, A—automated dispenser). Temperatures recorded in each sampling point $(\mathrm{M}-$ maturing mud tank, $\mathrm{P}$-peloid, $\mathrm{R}$-regenerating used mud) are also reported for the spring (SS) and autumn sampling (AS). Average temperature for each mud type, minimum and maximum values and first (Q1), second (Q2) and third quartile (Q3) are also reported.

\begin{tabular}{|c|c|c|c|c|c|c|c|c|}
\hline \multirow{2}{*}{ ID } & \multirow{2}{*}{$\begin{array}{c}\text { Cultivation } \\
\text { Plant Typology }\end{array}$} & \multirow{2}{*}{$\begin{array}{l}\text { Thermal Mud } \\
\text { Distribution }\end{array}$} & \multicolumn{3}{|c|}{ SS Temperature $\left({ }^{\circ} \mathrm{C}\right)$} & \multicolumn{3}{|c|}{ AS Temperature $\left({ }^{\circ} \mathrm{C}\right)$} \\
\hline & & & $\mathbf{M}$ & $\mathbf{P}$ & $\mathbf{R}$ & $\mathbf{M}$ & $\mathbf{P}$ & $\mathbf{R}$ \\
\hline 1 & $\mathrm{~T}$ & $\mathrm{D}$ & 32.8 & 55.1 & 43.5 & 24.0 & 59.0 & 42.0 \\
\hline 2 & $\mathrm{~T}$ & $\mathrm{D}$ & 44.5 & 40.1 & 47.0 & 55.0 & 66.0 & 48.0 \\
\hline 3 & $\mathrm{H}$ & A & 42.3 & 37.4 & 47.3 & 50.0 & 30.0 & 21.0 \\
\hline 4 & $\mathrm{H}$ & A & 49.4 & 39.0 & 30.1 & 48.0 & 25.0 & 24.0 \\
\hline 5 & $\mathrm{~T}$ & B & 23.0 & 54.5 & 45.7 & 20.0 & 59.0 & 20.0 \\
\hline 6 & $\mathrm{~T}$ & B & 48.7 & 58.7 & 41.0 & 43.0 & 58.0 & 35.0 \\
\hline 7 & $\mathrm{~T}$ & B & 52.4 & 33.0 & 51.1 & 52.0 & 64.0 & 42.0 \\
\hline 8 & $\mathrm{~T}$ & B & 36.7 & 56.5 & 44.8 & 42.0 & 58.0 & 46.0 \\
\hline 9 & $\mathrm{~T}$ & $\mathrm{D}$ & 32.3 & 56.3 & 50.3 & 36.0 & 51.0 & 43.0 \\
\hline 10 & $\mathrm{~T}$ & $\mathrm{D}$ & 47.3 & 56.8 & 50.4 & 44.0 & 52.0 & 51.0 \\
\hline 11 & $\mathrm{~T}$ & B & 47.4 & 48.9 & 43.6 & 59.0 & 62.0 & 53.0 \\
\hline 12 & $\mathrm{~T}$ & $\mathrm{D}$ & 41.0 & 56.5 & 49.1 & 50.0 & 61.0 & 40.0 \\
\hline 13 & $\mathrm{~T}$ & $\mathrm{D}$ & 40.6 & 59.0 & 60.2 & 45.0 & 48.0 & 46.0 \\
\hline 14 & $\mathrm{~T}$ & B & 58.6 & 60.1 & 43.8 & 45.0 & 62.0 & 49.0 \\
\hline 15 & $\mathrm{~T}$ & $\mathrm{D}$ & 40.5 & 57.6 & 31.5 & 34.0 & 58.0 & 57.0 \\
\hline 16 & $\mathrm{~T}$ & B & 69.2 & 67.3 & 55.0 & 52.0 & 63.0 & 62.0 \\
\hline 17 & $\mathrm{~T}$ & B & 57.3 & 58.6 & 45.0 & 33.0 & 59.0 & 50.0 \\
\hline 18 & $\mathrm{~T}$ & B & 41.2 & 56.7 & 58.4 & 54.0 & 57.0 & 52.0 \\
\hline 19 & $\mathrm{~T}$ & B & 36.2 & 47.8 & 40.1 & 42.0 & 57.0 & 43.0 \\
\hline 20 & $\mathrm{~T}$ & B & 51.1 & 62.0 & 60.6 & 45.0 & 56.0 & 49.0 \\
\hline 21 & $\mathrm{~T}$ & B & 58.5 & 56.5 & 56.3 & 42.0 & 70.0 & 45.0 \\
\hline 22 & $\mathrm{~T}$ & B & 48.0 & 66.9 & 56.4 & 46.0 & 60.0 & 45.0 \\
\hline 23 & $\mathrm{~T}$ & $\mathrm{D}$ & 42.9 & 59.6 & 64.8 & 39.0 & 57.0 & 28.0 \\
\hline 24 & $\mathrm{~T}$ & B & 56.7 & 62.6 & 45.5 & 42.0 & 64.0 & 54.0 \\
\hline 25 & $\mathrm{~T}$ & $\mathrm{D}$ & 52.7 & 49.3 & 42.6 & 35.0 & 40.0 & 45.0 \\
\hline 26 & $S$ & A & 72.0 & 27.0 & 27.0 & 66.0 & 54.0 & 60.0 \\
\hline 27 & $\mathrm{~T}$ & $\mathrm{D}$ & 49.1 & 71.2 & 86.6 & 31.0 & 70.0 & 43.0 \\
\hline 28 & $\mathrm{~T}$ & $\mathrm{D}$ & 38.0 & 55.0 & 45.0 & 55.0 & 58.0 & 41.0 \\
\hline 29 & $S$ & A & 45.0 & 50.0 & 52.0 & 45.0 & 56.0 & 41.0 \\
\hline \multirow[t]{7}{*}{30} & $\mathrm{~T}$ & B & 61.0 & 65.0 & 59.0 & 50.0 & 61.0 & 38.0 \\
\hline & & average & 47.2 & 54.2 & 49.1 & 44.1 & 56.5 & 43.8 \\
\hline & & $\min$ & 23.0 & 27.0 & 27.0 & 20.0 & 25.0 & 20.0 \\
\hline & & $\max$ & 72.0 & 71.2 & 86.6 & 66.0 & 70.0 & 62.0 \\
\hline & & Q1 & 40.7 & 49.5 & 43.7 & 39.8 & 56.0 & 41.0 \\
\hline & & Q2 & 47.4 & 56.5 & 47.2 & 45.0 & 58.0 & 45.0 \\
\hline & & Q3 & 52.6 & 59.5 & 56.0 & 50.0 & 61.8 & 49.8 \\
\hline
\end{tabular}

\subsection{Microbiological Analysis}

On the whole, $180 \mathrm{TM}$ samples were processed. Total viable counts of SS and AS for all mud typologies and incubation temperatures are reported in Table 3. 
Table 3. Total viable counts (TVCs). Microbial counts at 22, 37 and $55^{\circ} \mathrm{C}$ are reported for spring sampling (SS) and autumn sampling (AS) for all three mud typologies ( $\mathrm{M}$ - maturing mud, $\mathrm{P}$-peloid, R-regenerating used mud). Due to the high variability registered among different samples (25-78,400 colony forming units (CFU)/g), TVC values are conveniently divided into three classes, each reprised by a white-to-grey gradient. Graphical ranges are: $<10^{3} \mathrm{CFU} / \mathrm{g}\left(22.6 \%\right.$ of samples), $10^{3}-10^{4} \mathrm{CFU} / \mathrm{g}$ (59.4\% of samples) and $>10^{4} \mathrm{CFU} / \mathrm{g}(18.0 \%$ of samples). Average CFU count, its minimum and maximum values and first (Q1), second (Q2) and third quartile (Q3) are also given.

\begin{tabular}{|c|c|c|c|c|c|c|c|c|c|c|c|c|c|c|c|c|c|c|}
\hline \multirow[t]{2}{*}{ ID } & \multicolumn{9}{|c|}{ Spring Sampling (CFU/g) } & \multicolumn{9}{|c|}{ Autumn Sampling (CFU/g) } \\
\hline & \multicolumn{3}{|c|}{$\operatorname{TVC} 22^{\circ} \mathrm{C}$} & \multicolumn{3}{|c|}{$\operatorname{TVC} 37^{\circ} \mathrm{C}$} & \multicolumn{3}{|c|}{ TVC $55^{\circ} \mathrm{C}$} & \multicolumn{3}{|c|}{$\operatorname{TVC} 22^{\circ} \mathrm{C}$} & \multicolumn{3}{|c|}{$\operatorname{TVC} 37^{\circ} \mathrm{C}$} & \multicolumn{3}{|c|}{ TVC $55^{\circ} \mathrm{C}$} \\
\hline 1 & 10,750 & 3150 & 2800 & 3950 & 3750 & 4550 & 550 & 1950 & 4050 & 8450 & 4900 & 7750 & 2340 & 3000 & 7800 & 29,200 & 21,000 & 28,000 \\
\hline 2 & 910 & 315 & 1845 & 855 & 420 & 1900 & 45 & 700 & 500 & 930 & 770 & 680 & 2050 & 1450 & 870 & 10,350 & 11,300 & 10,700 \\
\hline 4 & 7350 & 6950 & 9900 & 11,350 & 8450 & 14,150 & 7250 & 6150 & 8350 & 24,000 & 27,400 & 17,000 & 15,200 & 8100 & 8300 & 12,700 & 8600 & 8850 \\
\hline 5 & 4400 & 2500 & 3100 & 4300 & 3250 & 5700 & 440 & 2650 & 5400 & 3500 & 7500 & 5700 & 7400 & 9300 & 7100 & 12,650 & 78,200 & 21,750 \\
\hline 6 & 6800 & 1805 & 1370 & 8050 & 1350 & 1425 & 17,300 & 745 & 5450 & 3750 & 3800 & 3100 & 3100 & 3000 & 2600 & 19,300 & 14,200 & 16,800 \\
\hline 7 & 3450 & 7100 & 1390 & 3850 & 5600 & 800 & 4700 & 5800 & 4500 & 25 & 6500 & 11,200 & 40 & 3800 & 680 & 185 & 16,500 & 13,300 \\
\hline 8 & 2490 & 2950 & 2385 & 2700 & 3450 & 1750 & 6250 & 3450 & 1590 & 8050 & 16,800 & 5800 & 6200 & 17,400 & 5450 & 21,100 & 32,400 & 17,200 \\
\hline 11 & 1005 & 1850 & 665 & 905 & 1195 & 985 & 5800 & 560 & 755 & 1480 & 3400 & 2200 & 2500 & 9000 & 3550 & 2850 & 14,400 & 1700 \\
\hline 12 & 235 & 985 & 740 & 770 & 1350 & 810 & 1105 & 1145 & 535 & 940 & 785 & 1460 & 1090 & 1400 & 2600 & 10,650 & 9400 & 46,800 \\
\hline 13 & 270 & 525 & 515 & 465 & 495 & 555 & & 570 & & 1150 & 575 & 715 & 800 & & 470 & 2350 & & 2700 \\
\hline 14 & 2800 & 2950 & 3350 & 3550 & 6150 & 7400 & 3150 & 4750 & 4800 & 2050 & 2900 & 13,500 & 2600 & 2650 & 16,600 & 4600 & 6300 & 11,650 \\
\hline 15 & 5400 & 5400 & 11,350 & 5050 & 6550 & 6350 & 7300 & 11,550 & 13,750 & 5300 & 2750 & 51,200 & 7750 & 5550 & 4450 & 78,400 & 51,200 & 43,200 \\
\hline 16 & 4150 & 340 & 465 & 2700 & 370 & 445 & 2250 & 240 & 340 & 2800 & 2350 & 1450 & 1900 & 1150 & 1650 & 2050 & 285 & 300 \\
\hline 17 & 3300 & 760 & 1630 & 3300 & 520 & 1165 & 4000 & 4250 & 1050 & 3300 & 1400 & 3050 & 2350 & 1020 & 6000 & 5500 & 19,000 & 55,200 \\
\hline 18 & 585 & 630 & 205 & 585 & & 270 & 732,5 & & & & & & & & & & 3400 & 3110 \\
\hline 19 & 2760 & 2610 & 27 & 3100 & 3250 & 3750 & 4000 & 2400 & 320 & 6400 & 2750 & 2750 & 4700 & 3500 & 4050 & 14,400 & 14,200 & 12,400 \\
\hline 26 & 420 & & 20 & 3 & 62 & 1705 & 2200 & & 1 & 37 & 14,220 & 15 & 860 & 98 & & 75 & 8400 & 5500 \\
\hline 27 & 2550 & 1740 & 19 & 530 & $1 \mathrm{~s}$ & 250 & 12,150 & 2000 & 2500 & 8300 & 2200 & 12,100 & 635 & 13 & 195 & 4650 & 1220 & 4350 \\
\hline 28 & 4810 & 4040 & 6240 & 470 & 610 & 640 & 12,400 & 8000 & 13,300 & 4600 & 6000 & 7550 & 470 & 575 & 410 & 21,450 & 20,550 & 25,400 \\
\hline 29 & 34,000 & 12,400 & 26,400 & & 13,700 & 93 & 3950 & & 5250 & 26,550 & 25,000 & 57,600 & 13,600 & 15,300 & 32, & 4800 & 5450 & 5800 \\
\hline 30 & 3800 & 3400 & 11,900 & 4300 & 6300 & 8700 & 1150 & 1350 & 3350 & 2060 & 7000 & 8700 & 930 & 4700 & 6100 & 535 & 5250 & 4950 \\
\hline average & 5361 & 3396 & 4522 & 3533 & 4762 & 4542 & 5239 & 4659 & 4861 & 4468 & 5879 & 9083 & 3319 & 5393 & 6401 & 12,789 & 15,942 & 15,229 \\
\hline $\min$ & 235 & 65 & 205 & 205 & 180 & 250 & 45 & 240 & 340 & 25 & 510 & 400 & 40 & 135 & 195 & 70 & 285 & 300 \\
\hline $\max$ & 34,600 & 17,450 & 26,400 & 17,550 & 51,800 & 38,750 & 22,600 & 38,300 & 30,500 & 26,550 & 27,400 & 57,600 & 15,200 & 18,800 & 41,600 & 78,400 & 78,200 & 55,200 \\
\hline Q1 & 934 & 1005 & 1370 & 771 & 543 & 998 & 762 & 845 & 1150 & 933 & 1458 & & 908 & 1213 & 1598 & 3363 & 5525 & 4163 \\
\hline Q2 & 3050 & 21 & 2223 & 32 & 20 & & 39 & & & & & & 19 & & & & 9700 & 11,175 \\
\hline Q3 & 5138 & 3880 & 4975 & 4713 & 5138 & 6188 & 7000 & 5500 & 5438 & 5263 & 6875 & 8775 & 4313 & 8775 & 6325 & 15,750 & 18,375 & 22,688 \\
\hline
\end{tabular}


Counts for total coliforms and E. coli are reported in Table 4. Total coliforms were isolated from 56 samples (31.1\%). In detail, total coliforms were found in $21 \mathrm{M}(11.7 \%), 16 \mathrm{P}(8.9 \%)$ and $19 \mathrm{R}$ (10.6\%) mud samples. E. coli was detected in 4 samples (2.8\%), of whom $2 \mathrm{M}(1.1 \%)$ and $2 \mathrm{R}(1.1 \%)$ samples respectively.

Table 4. Total coliforms and E. coli. Microbial counts are reported for both indicator parameters. The first number refers to total coliforms whilst the one in round brackets to E. coli colonies. $\mathrm{M}-$ maturing mud, $\mathrm{P}$ - peloid, R-regenerating used mud.

\begin{tabular}{|c|c|c|c|c|c|c|}
\hline \multirow[b]{3}{*}{ ID } & \multicolumn{6}{|c|}{ Total Coliforms and Escherichia coli (CFU/g) } \\
\hline & \multicolumn{3}{|c|}{ Spring Sampling } & \multicolumn{3}{|c|}{ Autumn Sampling } \\
\hline & $\mathbf{M}$ & $\mathbf{P}$ & $\mathbf{R}$ & $\mathbf{M}$ & $\mathbf{P}$ & $\mathbf{R}$ \\
\hline 1 & $1120(85)$ & 5 & $25(20)$ & 68 & $<1$ & 191 \\
\hline 2 & $<1$ & $<1$ & $<1$ & $<1$ & $<1$ & $<1$ \\
\hline 3 & $<1$ & 10 & $<1$ & $<1$ & 62 & 50 \\
\hline 4 & 28 & 1 & 17 & 21 & 176 & 20 \\
\hline 5 & 73 & 1 & 5 & $16(2)$ & 18 & 312 \\
\hline 6 & $<1$ & $<1$ & $<1$ & $<1$ & $<1$ & $<1$ \\
\hline 7 & 2 & 26 & $<1$ & $<1$ & $<1$ & 8 \\
\hline 8 & $<1$ & $<1$ & $<1$ & 5 & $<1$ & 6 \\
\hline 9 & 66 & $<1$ & $<1$ & $<1$ & $<1$ & $<1$ \\
\hline 10 & $<1$ & $<1$ & $<1$ & $<1$ & $<1$ & $<1$ \\
\hline 11 & $<1$ & $<1$ & $<1$ & $<1$ & 2 & $<1$ \\
\hline 12 & $<1$ & $<1$ & $<1$ & $<1$ & 2 & $<1$ \\
\hline 13 & $<1$ & $<1$ & $<1$ & $<1$ & $<1$ & $<1$ \\
\hline 14 & $<1$ & 2 & $<1$ & 70 & $<1$ & 48 \\
\hline 15 & 2 & $<1$ & $2(24)$ & 16 & 2 & 26 \\
\hline 16 & $<1$ & $<1$ & $<1$ & $<1$ & $<1$ & $<1$ \\
\hline 17 & $<1$ & $<1$ & $<1$ & $<1$ & $<1$ & $<1$ \\
\hline 18 & $<1$ & $<1$ & $<1$ & $<1$ & $<1$ & $<1$ \\
\hline 19 & $<1$ & 4 & $<1$ & 10 & $<1$ & 2 \\
\hline 20 & $<1$ & $<1$ & $<1$ & $<1$ & $<1$ & $<1$ \\
\hline 21 & $<1$ & $<1$ & $<1$ & 88 & $<1$ & 26 \\
\hline 22 & $<1$ & $<1$ & $<1$ & $<1$ & $<1$ & $<1$ \\
\hline 23 & $<1$ & $<1$ & $<1$ & 96 & 2 & $<1$ \\
\hline 24 & $<1$ & $<1$ & $<1$ & $<1$ & $<1$ & $<1$ \\
\hline 25 & $<1$ & $<1$ & $<1$ & $<1$ & $<1$ & $<1$ \\
\hline 26 & 17 & 1471 & 1020 & 4 & $<1$ & 14 \\
\hline 27 & $<1$ & $<1$ & $<1$ & 100 & $<1$ & 4 \\
\hline 28 & 6 & $<1$ & $<1$ & $<1$ & $<1$ & 6 \\
\hline 29 & 2 & 12 & $<1$ & 14 & $<1$ & 22 \\
\hline 30 & $<1$ & $<1$ & $<1$ & $<1$ & $<1$ & $<1$ \\
\hline
\end{tabular}

Counts for enterococci are reported in Table 5. Enterococci were found in 132 (73.3\%) samples, with the same frequency $(24.4 \%)$ among $\mathrm{M}, \mathrm{P}$ and $\mathrm{R}$ samples. 
Table 5. Enterococci. Microbial counts are reported for the enterococci indicator parameter. $\mathrm{M}-$ maturing mud, $\mathrm{P}$ - peloid, $\mathrm{R}$-regenerating used mud.

\begin{tabular}{|c|c|c|c|c|c|c|}
\hline \multirow[b]{3}{*}{ ID } & \multicolumn{6}{|c|}{ Enterococci (CFU/g) } \\
\hline & \multicolumn{3}{|c|}{ Spring Sampling } & \multicolumn{3}{|c|}{ Autumn Sampling } \\
\hline & $\mathbf{M}$ & $\mathbf{P}$ & $\mathbf{R}$ & $\mathbf{M}$ & $\mathbf{P}$ & $\mathbf{R}$ \\
\hline 1 & 145 & 15 & 20 & 177 & 206 & 269 \\
\hline 2 & $<1$ & $<1$ & $<1$ & 30 & 90 & $<1$ \\
\hline 3 & $<1$ & 5 & $<1$ & $<1$ & 27 & 170 \\
\hline 4 & 16 & 13 & 52 & 90 & 94 & 60 \\
\hline 5 & $<1$ & $<1$ & 25 & 2 & 6 & $<1$ \\
\hline 6 & 41 & 14 & 18 & 8 & 2 & 8 \\
\hline 7 & 208 & 24 & 288 & $<1$ & 72 & 92 \\
\hline 8 & 16 & 30 & 18 & 22 & 32 & 10 \\
\hline 9 & 20 & 22 & 30 & 56 & 10 & 12 \\
\hline 10 & 2 & 2 & 10 & 8 & $<1$ & 4 \\
\hline 11 & 2 & 66 & 36 & 14 & $<1$ & $<1$ \\
\hline 12 & $<1$ & $<1$ & $<1$ & 14 & 6 & 10 \\
\hline 13 & $<1$ & $<1$ & $<1$ & 2 & 2 & $<1$ \\
\hline 14 & 2 & 4 & $<1$ & $<1$ & 4 & $<1$ \\
\hline 15 & 16 & 8 & 14 & 8 & 6 & 16 \\
\hline 16 & $<1$ & $<1$ & $<1$ & 2 & $<1$ & 2 \\
\hline 17 & 14 & $<1$ & 2 & 10 & 4 & 38 \\
\hline 18 & $<1$ & $<1$ & $<1$ & 2 & 4 & $<1$ \\
\hline 19 & 14 & 17 & 32 & 12 & 18 & 32 \\
\hline 20 & 40 & 34 & 8 & $<1$ & $<1$ & $<1$ \\
\hline 21 & 6 & 16 & 14 & 26 & 6 & 4 \\
\hline 22 & 10 & 8 & 4 & 2 & $<1$ & 8 \\
\hline 23 & 14 & 48 & 42 & 4 & $<1$ & 24 \\
\hline 24 & 40 & 20 & 126 & 22 & 38 & 26 \\
\hline 25 & 1 & 7 & 9 & $<1$ & 14 & 2 \\
\hline 26 & 69 & 44 & 62 & 26 & 28 & 12 \\
\hline 27 & 12 & 2 & 35 & 40 & $<1$ & 6 \\
\hline 28 & 106 & 56 & 72 & 38 & 18 & 86 \\
\hline 29 & $<1$ & 8 & $<1$ & $<1$ & $<1$ & 28 \\
\hline 30 & $<1$ & 4 & $<1$ & $<1$ & $<1$ & 4 \\
\hline
\end{tabular}

Among assessed parameters, anaerobic sulfite-reducing clostridia resulted the most abundant group (Table 6): growth was observed in 166 (92.2\%) samples, with CFU counts ranging from 3 to $2070 \mathrm{CFU} / \mathrm{g}$. 
Table 6. Sulfite-reducing clostridia. Microbial counts are reported. Similarly to the partition done for TVCs, $53.9 \%$ of clostridia counts are in the $<100 \mathrm{CFU} / \mathrm{g}$ class (white background), $40.6 \%$ in the 100-1000 CFU/g (light grey) and 5.6\% in the $>1000 \mathrm{CFU} / \mathrm{g}$ one (dark grey). M-maturing mud, $\mathrm{P}$-peloid, R-regenerating used mud.

\begin{tabular}{|c|c|c|c|c|c|c|}
\hline \multirow[b]{3}{*}{ ID } & \multicolumn{6}{|c|}{ Sulfite-Reducing Clostridia (CFU/g) } \\
\hline & \multicolumn{3}{|c|}{ Spring Sampling } & \multicolumn{3}{|c|}{ Autumn Sampling } \\
\hline & $\mathbf{M}$ & $\mathbf{P}$ & $\mathbf{R}$ & $\mathbf{M}$ & $\mathbf{P}$ & $\mathbf{R}$ \\
\hline 1 & 945 & 127 & 1635 & 300 & 395 & 590 \\
\hline 2 & 135 & 30 & 170 & 54 & 34 & 28 \\
\hline 3 & 55 & 395 & 1095 & $<1$ & 160 & 740 \\
\hline 4 & 1780 & 1535 & 2070 & 520 & 285 & 140 \\
\hline 5 & 290 & 48 & 300 & 680 & 115 & 1525 \\
\hline 6 & 480 & 95 & 240 & 300 & 15 & 35 \\
\hline 7 & 520 & 350 & 112 & $<1$ & 10 & 40 \\
\hline 8 & 130 & 42 & 58 & 100 & 75 & 45 \\
\hline 9 & 20 & 85 & 20 & 1220 & 120 & 170 \\
\hline 10 & 35 & 10 & 110 & 44 & 4 & 24 \\
\hline 11 & 10 & 40 & 16 & $<1$ & $<1$ & 13 \\
\hline 12 & 44 & 220 & 160 & 22 & 16 & 120 \\
\hline 13 & 25 & 20 & 35 & 20 & 120 & 20 \\
\hline 14 & $<1$ & 90 & 20 & 55 & 115 & 245 \\
\hline 15 & 685 & 1045 & 335 & 450 & 225 & 65 \\
\hline 16 & 350 & 25 & 5 & 85 & $<1$ & 10 \\
\hline 17 & 250 & 35 & 80 & $<1$ & 20 & 25 \\
\hline 18 & 35 & 10 & 5 & $<1$ & 40 & 60 \\
\hline 19 & 295 & 935 & 560 & 25 & 20 & 60 \\
\hline 20 & 510 & 120 & 1380 & 290 & 120 & $<1$ \\
\hline 21 & 175 & 290 & 380 & 120 & 110 & 60 \\
\hline 22 & 80 & $<1$ & 95 & 60 & 17 & 24 \\
\hline 23 & 860 & 185 & 345 & 40 & 690 & 230 \\
\hline 24 & 20 & 125 & 145 & 165 & 210 & 270 \\
\hline 25 & 15 & 80 & 340 & $<1$ & 30 & 5 \\
\hline 26 & $<1$ & 140 & 1095 & $<1$ & 360 & 320 \\
\hline 27 & 14 & 3 & 10 & 95 & 7 & 18 \\
\hline 28 & 114 & 99 & 52 & 72 & 14 & 32 \\
\hline 29 & 595 & 240 & 920 & 95 & 180 & 210 \\
\hline 30 & 85 & 95 & 160 & $<1$ & 15 & 75 \\
\hline
\end{tabular}

Table 7 jointly reports the few samples in which $P$. aeruginosa or dermatophytes were retrieved. $P$. aeruginosa was found in 2 (1.1\%) AS samples, whereas dermatophytes in 5 SS samples of R mud. S. aureus was not found in any of the processed samples. 
Table 7. Positive samples for Pseudomonas aeruginosa or dermatophytes. Dermatophytes were found in regenerating mud samples only. AS—autumn sampling; SS—-spring sampling; M-maturing mud, $\mathrm{P}$-peloid, R—regenerating used mud; CFU—colony forming units.

\begin{tabular}{ccc}
\hline & ID & CFU/g \\
\hline P. aeruginosa & AS-4P & 40 \\
\hline Dermatophytes & SS-1R & 100 \\
\hline & SS-3R & 5 \\
\hline & SS-4R & 2 \\
\hline & SS-18R & 2 \\
\hline & SS-26R & 10 \\
\hline
\end{tabular}

\subsection{Statistical Analysis}

Comparison of thermal water temperatures recorded during SS and AS returns a significant difference for R tanks only ( $p$-value $=0.041 ; \mathrm{W}$-statistic $=133$ ), with an average temperature delta of $-5{ }^{\circ} \mathrm{C}$ during AS. As suggested by raw data, TVCs generally achieve higher counts during AS. Consistently, significant difference between SS and AS is found for $55^{\circ} \mathrm{C}$ TVCs of M, P and R mud samples. In addition, the $22{ }^{\circ} \mathrm{C}$ TVCs for $\mathrm{P}$ and $\mathrm{R}$ samples also achieves statistical significance, with higher values during AS (Table 8).

Table 8. Comparison of TVCs of mud samples between SS and AS. The table provides $p$-values and W statistics for the Wilcoxon signed rank test. Significant $p$-values $(<0.05)$ are marked with asterisk $\left(^{*}\right)$. $\mathrm{M}$-maturing mud, $\mathrm{P}$-peloid, $\mathrm{R}$-regenerating used mud.

\begin{tabular}{cccc}
\hline & & $p$-Value & W Stat \\
\hline $\mathrm{M}$ & 22 & 0.158 & 141.0 \\
\hline & 37 & 0.905 & 212.0 \\
\hline & 55 & $0.002 *$ & 83.0 \\
\hline $\mathrm{P}$ & 22 & $0.020^{*}$ & 119.0 \\
\hline & 37 & 0.225 & 173.5 \\
\hline & 55 & $0.000 *$ & 43.0 \\
\hline $\mathrm{R}$ & 22 & $0.026^{*}$ & 125.0 \\
\hline & 37 & 0.102 & 153.0 \\
\hline & 55 & $<0.001 *$ & 32.0 \\
\hline
\end{tabular}

Significant correlation between TVC and temperature was found only for AS samples. In detail, TVCs at 22,37 and $55{ }^{\circ} \mathrm{C}$ for $\mathrm{M}$ as well as at $22{ }^{\circ} \mathrm{C}$ and $55^{\circ} \mathrm{C}$ for $\mathrm{R}$ achieved statistical significance (Table 9). Basically, the higher the temperature the lower the TVC, as hinted by negative $\rho$ coefficients.

The assessment of temperature influence on presence/absence of indicator parameters other than TVCs, returned statistical significance for total coliforms (M, P and R samples), E. coli (M samples) and enterococci (R samples) (Table 10). Average thermal water temperature was calculated for presence and absence groups of significant parameters, suggesting that presence always occurred with lower temperatures (Table 11). 
Table 9. Correlation between thermal water temperature and TVCs of AS mud samples. The table provides $p$-values and $\rho$ coefficients for Spearman's R test. Significant $p$-values $(<0.05)$ are marked with asterisk $\left(^{*}\right)$. AS—autumn sampling; $\mathrm{M}$-maturing mud, $\mathrm{P}$ - peloid, $\mathrm{R}$-regenerating used mud.

\begin{tabular}{ccccc}
\hline Sampling & Mud Type & Growth Temp. $\left({ }^{\circ} \mathrm{C}\right)$ & $p$-Value & $\rho$ \\
\hline AS & 22 & $0.027^{*}$ & -0.404 \\
\hline & $\mathrm{M}$ & 37 & $0.025^{*}$ & -0.408 \\
\hline AS & & 55 & $0.037^{*}$ & -0.383 \\
\hline & $\mathrm{P}$ & 22 & 0.333 & -0.183 \\
\hline & & 37 & 0.209 & -0.236 \\
\hline AS & & 55 & 0.441 & -0.146 \\
\hline & $\mathrm{R}$ & 22 & $0.010 *$ & -0.464 \\
\hline & & 37 & 0.121 & -0.289 \\
\hline
\end{tabular}

Table 10. Mann-Whitney U test for indicator parameter presence/absence in mud samples. $p$-values and $U$ statistics are reported for the Mann-Whitney $U$ test. All $p$-values are significant $(<0.05)$ and thus are marked with asterisk $\left(^{*}\right)$. $\mathrm{M}$-maturing mud, $\mathrm{P}$-peloid, $\mathrm{R}$-regenerating used mud.

\begin{tabular}{cccc}
\hline Mud Type & Indicator Parameter & $p$-Value & $\mathbf{U}$ \\
\hline \multirow{2}{*}{$\mathbf{M}$} & Total coliforms & $0.006^{*}$ & 233.0 \\
\cline { 2 - 4 } & E. coli & $0.026^{*}$ & 4 \\
\hline $\mathbf{U}$ & Total coliforms & $0.005^{*}$ & 182.5 \\
\hline \multirow{2}{*}{$\mathbf{R}$} & Total coliforms & $0.001^{*}$ & 184.0 \\
\cline { 2 - 4 } & Enterococci & $0.022^{*}$ & 214.5 \\
\hline
\end{tabular}

Table 11. Presence/absence of indicator parameters and average thermal water temperature. Average temperature (AT) for positive and negative samples is reported. Presence of indicator parameters ( $>0 \mathrm{CFU} / \mathrm{g}$ ) always occurred in samples with an average lower thermal water temperature. $\mathrm{M}-$ maturing mud, $\mathrm{P}$ - peloid, $\mathrm{R}$-regenerating used mud.

\begin{tabular}{cccc}
\hline Mud Type & Indicator Parameter & AT $\left({ }^{\circ} \mathbf{C}\right)$ Presence & AT $\left({ }^{\circ} \mathbf{C}\right)$ Absence \\
\hline $\mathbf{M}$ & total coliforms & 41.1 & 48.1 \\
\hline $\mathbf{P}$ & E. coli & 39.6 & 46.0 \\
\hline $\mathbf{R}$ & total coliforms & 47.2 & 58.3 \\
\hline & total coliforms & 39.6 & 49.6 \\
\hline
\end{tabular}

\section{Discussion}

The relevance of microbiological hygiene quality has long been neglected for TMs and literature addressing this topic is still quite limited. Sanchez-Espejo et al. [30] evaluated the microbiological compliance of five raw clay samples used to prepare TMs with the limits proposed by the European regulations for medicinal products. Free-living pathogenic amoebas were sought in mud samples by Scaglia et al. [29]. "Disappearance of pathogens" after maturation was reported by Galzigna et al., but no methods nor numeric results were provided [28]. Tentatively, a study from Turkey addressed the microbial contamination of TMs, but methods and results are ambiguous and reproducibility is rather penalized [31]. Quintela et al. [32] published a pilot study on the microbiological quality of maturated volcanic muds: they evaluated the total microbial count at 22 and $37^{\circ} \mathrm{C}$ by pour-plate 
method and total coliforms by membrane filtration technique. In another work they also succinctly discussed, but didn't assess, microbial content of TMs, suggesting to take into account some indicator parameters, i.e., enterobacteria, Streptococcus, E. coli and total and fecal coliforms, to ensure safety of patients [3].

Evaluation of microbiological hygiene quality of TMs carried out in the present study encompasses a precise knowledge of both maturation process and pelotherapy protocol, so as to ensure a comprehensive assessment of the whole mud cultivation chain and its critical points. Surveyed facilities are located in the Euganean Thermal District, Veneto Region, NE Italy. This territory is one the oldest thermal areas in Europe and boasts an ancient pelotherapy tradition. In the Euganean thermal basin, virgin mud for pelotherapy is exclusively drawn from two mining concessions, i.e., the Lispida ( $\left.\mathrm{N} 45^{\circ} 16^{\prime} 41^{\prime \prime} \mathrm{E} 11^{\circ} 46^{\prime} 13^{\prime \prime}\right)$ and the (N 45 $16^{\prime} 11^{\prime \prime}$ E $\left.11^{\circ} 44^{\prime} 37^{\prime \prime}\right)$ Arquà Lakes. Currently, a maximum amount of $1000 \mathrm{~m}^{3}$ can be extracted per year, as virgin mud is a non-renewable resource [18]. Thermal facilities are thus forced to reuse TM for multiple therapeutic applications. By law, virgin mud must be independently matured by each pelotherapy facility.

Maturation of virgin mud lasts several months, during which the mud is submerged with thermal water. The first hygienic issue concerns the presence of undesirable microorganisms in maturing mud, as the result of a naturally-occurring contamination of the extraction lake sediments. Optimal maturation of TMs in terms of bioglea growth and production of therapeutic compounds should be carried out in the $30-42{ }^{\circ} \mathrm{C}$ temperature range [33]. Registered temperatures in $\mathrm{M}$ sampling sites were compared to the suggested range. A low compliance was generally observed (i.e., about $30 \%$ of facilities), since maturing muds were mostly kept at $>42{ }^{\circ} \mathrm{C}$. Although affecting the maturation process, this should not penalize hygienic aspects but rather supporting the elimination of undesired environmental microorganisms.

Once maturation is achieved, TMs are traditionally "pasteurized" with thermal water for about 24-48 $\mathrm{h}$ prior to therapy [33]. Microbiological analysis of ready-to-use TMs is thus indicative of the efficacy of this intended pre-therapy sanification. Pasteurization of TMs in surveyed facilities was carried out by $75 \%$ of facilities at a temperature of $\leq 60^{\circ} \mathrm{C}$.

As mentioned, due to the locally enforced TM extraction limit [18], all surveyed facilities re-use TMs for multiple treatments. The hygienic concern relevant to this step, regards the possible transfer of microorganisms from the patient's skin to the applied mud. After treatment, the used mud is immediately transferred in a dedicated collection tank filled with thermal water. The regeneration step lasts about three days. Since regeneration will be followed by a new maturation cycle, correct management of this step, in terms of thermal water temperature, is essential to avoid persistence of microbial contaminants throughout the cultivation process. By the way, only six facilities employed water $\geq 60^{\circ} \mathrm{C}$ amidst SS and AS. Moreover, statistical comparison of thermal water temperatures between the two sampling campaigns suggests that $\mathrm{R}$ temperatures were significantly lower during AS (average difference $=-5^{\circ} \mathrm{C}$ ), probably due to the enhanced cooling of thermal water during autumn environmental conditions.

Microbiological analysis of environmental samples usually employs indicator parameters to evaluate the overall quality or potential contamination of samples: contextualization of selected indicator parameters and interpretation of obtained results is hereby presented.

Total viable count is a generic indicator parameter representative for the broad mud microbial colonization, and it is essentially unrelated to pathogen species. TVCs highlighted a copious microbial growth in all samples. Significantly higher TVC counts were observed during AS, if compared to SS. Lower thermal water temperatures reported for $\mathrm{R}$ tanks during AS could explain this finding, but still a similar explanation can't be invoked for M and P samples. Supposedly, environmental variables other than thermal water temperature (e.g., sunlight, air temperature, rainfall) are capable of influencing microbial growth, but an in-depth understanding of such microbial ecological dynamics falls outside the scope of the present research. 
Total coliforms encompass a broad class of bacteria commonly found in the natural environment, whereas E. coli stands for recent fecal contamination. Total coliforms in M samples should thus not come unexpected. Virgin mud most likely carries coliforms as natural colonizers that further survive in the cultivation plant thanks to the permissive thermal water temperature of maturation tanks. Two M samples (1M-SS; 5M-AS) were found positive also for E. coli. Its presence could depend once more on pristine contamination of virgin mud or either on a faulty regeneration procedure of the used mud. Actually, it was impossible to determine whether sampled $\mathrm{M}$ mud was virgin mud or regenerated mud. Consistently, temperatures of both $\mathrm{M}$ and $\mathrm{R}$ sampling sites were quite low for the two facilities (Table 2). Hygienic quality of $P$ samples is of core importance. Whereas presence of total coliforms in P samples can still be tolerated, absence of E. coli should be required. Among the analyzed mature mud samples, no one resulted contaminated by E. coli. Consistently, presence of $E$. coli in TMs can be prevented by pasteurization with a correct thermal water temperature and contact time. E. coli was found in $2 \mathrm{R}$ samples as well (1R-SS with $20 \mathrm{CFU} / \mathrm{g} ; 15 \mathrm{R}-\mathrm{SS}$ with $24 \mathrm{CFU} / \mathrm{g}$. Thermal water in involved facilities once more was too cold to ensure proper sanification of used mud (Table 2). Similarly to coliforms, the presence of enterococci in natural muds is quite predictable and acceptable. Several studies pointed out how lake sediments are significant reservoirs of enterococci [34]. Members of the Staphylococcus genus can be either saprophytic environmental species or microorganisms transferred from the patient's skin, so that peculiar attention was paid to the possible presence of opportunistic pathogen species S. aureus in P and R samples. Nevertheless, no sample resulted positive for this indicator. Among the investigated parameters, anaerobic clostridia, which are commonly found in the environment, represented the most abundant bacterial class. Mud is per se an anoxic matrix that favors the proliferation of clostridia and their variable abundance is probably influenced by the mud cultivation and mixing procedures implemented by each facility. P. aeruginosa was isolated in two samples (4P-SS and 9M-SS). Consistently, temperature registered for sample $4 \mathrm{P}$ and $9 \mathrm{M}$ was unsuitable for sanitization purposes (Table 2). Skin-disease causing dermatophyte fungi were sporadically isolated in $5 \mathrm{R}$ samples. Their most plausible origin is direct transfer from patient's skin to the used mud.

Statistical analysis supports the relevance of thermal water temperature on microbial growth of indicator parameters. Presence or absence of total coliforms resulted significantly linked to different temperature clusters, as shown in Table 11. Similarly, M samples with E. coli and R samples with enterococci significantly correlate to lower average temperatures. Although statistical significance was achieved only by the above discussed samples, a similar trend can be observed for all considered indicators and in all mud typologies, confirming the critical role of temperature on the hygienic profiling of TMs.

Overall, some of the selected indicator parameters proved suitable for the assessment of microbiological hygienic quality of TMs. Nevertheless, the need of a precise thermal water temperature guideline value for each mud cultivation phase emerged as a critical issue. A reference protocol addressing both the optimization of cultivation temperatures and setting microbiological quality requirements can now be ventured. During maturation, priority should be given to the correct growth of the bioglea, so that TMs can achieve the best therapeutic quality. A temperature range of $30-42{ }^{\circ} \mathrm{C}$ is therefore recommended. Temperatures $>42{ }^{\circ} \mathrm{C}$ are strongly discouraged since, although enhancing precocious mud sanitization, they can seriously compromise the correct maturation of TMs. Hygienic implications effectively come into play in the pre-therapy pasteurization step. It should be desirable for thermal water in $\mathrm{P}$ tanks to be at $60-65^{\circ} \mathrm{C}$. Of future interest, the multidisciplinary evaluation of a temperature range with an upper limit, so as to grant sanification without irretrievably denaturing therapeutic compounds. Furthermore, it should be compulsory to thoroughly sanitize the used mud before they start a new maturation cycle. Thermal water in regeneration tanks should therefore be kept at a temperature of $\geq 65^{\circ} \mathrm{C}$, for at least $72 \mathrm{~h}$. Disruption of therapeutic compounds and of bio-active microorganisms should not be feared in this case, since the use of a "starter" bioglea can efficiently promote a new maturation process [33]. Of course, since only cultivation techniques of the Euganean basin were considered in the present study, a limitation of the above proposal is that it should be 
calibrated for other pelotherapy districts. However, the general assessment of hygiene-related critical points of the mud cultivation chain is transferable to other pelotherapy operating facilities.

To the author's knowledge, there is no national nor international legislation specifically addressing microbial requirements for thermal muds and peloids. Cosmetics regulations can possibly share some similarity, if considering the clayey nature of peloids. The European Regulation (EC) No. 1223/2009 recommends peculiar attention to microbiological purity of topical products to be used on mucous membranes in general, on damaged skin. It also stresses the importance of microbiological quality of products, if used by immunocompromised persons or by elderly people, in reason of their physiological immunosenescence. Safety issues resulting from microbiologically contaminated topical products are sporadic (e.g., infections caused by Gram-negative organisms), yet not negligible [35,36]. Similar recommendations could also suit peloids, especially if taking into account the typical pelotherapy applications and the elderly patients. Nevertheless, hygienic quality of thermal muds should not only focus on raw materials (i.e., virgin mud) and the "finished product" (i.e., the ready-for-therapy peloid). Quality of regenerated muds should definitely be granted by all facilities that reuse them for multiple pelotherapy applications on different patients. Of course thermal muds and peloids need not to be sterile, but they certainly should not be contaminated with undesired or potentially pathogenic microorganisms.

Among the assessed indicator parameters, some resulted not informative in respect of the hygienic quality characterization of TMs. In detail, TVCs and clostridia resulted too numerically variable among different samples to provide a reliable reference index; total coliforms and enterococci were commonly found as part of the environmental microflora. Recommended indicator parameters and guideline values thus are: absence (i.e., 0 CFU/g) of E. coli, Pseudomonas aeruginosa, Staphylococcus aureus and dermatophytes. Moreover, microbiological hygiene requirements can be reasonably demanded only for ready-for-therapy TMs, after a proper pasteurization step is carried out.

In the end, some considerations about the methodological choices are shared. The present study adopted a classic bacteriology approach after considering some pragmatic and scope-determined aspects. In the first place, a large number of mud samples (i.e., 180) had to be processed, so that the affordability of culture methods certainly played a role. Moreover, culture methods provide precious quantitative details and evaluate the microorganism vitality, a data crucially relevant for a complete risk assessment. Molecular methods such as microarrays or next generation sequencing (e.g., [27,37]) were considered to provide analytical details even beyond the core aim of this pilot research.

\section{Conclusions}

Microbial hygienic quality of TMs was thoroughly assessed after the validation of an ad hoc laboratory method. Analysis of TM samples (i.e., maturing mud, peloids and used mud) highlighted how presence of undesired microorganisms can either result from environmental contamination or transfer from patients' skin. A core set of suitable indicator parameters for evaluating the microbiological hygiene quality of TMs could encompass Escherichia coli, Pseudomonas aeruginosa, Staphylococcus aureus and dermatophytes. Absence ( $0 \mathrm{CFU} / \mathrm{g}$ ) of such indicators is recommended. Proper management of thermal water temperatures throughout the diverse phases of the mud cultivation process represents a critical issue. If, on the one hand, maturation of virgin mud must ensure the achievement of the best therapeutic properties, on the other a pasteurization step of TMs with thermal water $\geq 60^{\circ} \mathrm{C}$ should be compulsory just before treatment, so as to grant its hygienic quality as well.

\section{Glossary of research terms}

In the present work, terms that belong to the field of thermal medicine adhere to the reference glossary proposed by Gomes et al. [2]. Additional voice is given by Authors for Regeneration. Concise definitions are hereby reported:

- Bioglea: biogenic gelatinous pellicle of yellowish, greenish, grayish or reddish colour, deposited in presence of sulfur-containing waters. 
- Maturation: the blending process of muds with thermal water, either in the natural environment or in artificial plants, during which maturing mud achieves therapeutic properties.

- Peloid: maturated mud with therapeutic properties.

- Pelotherapy: external application of thermal muds for therapeutic purposes.

- Regeneration: sanitization process of used thermal muds. It is implemented before a new maturation cycle starts by facilities that use muds for multiple pelotherapy applications on different patients.

Author Contributions: T.B.: Conceptualization, funding acquisition, methodology, writing-Review and editing. I.A.: investigation, methodology, formal analysis, writing-Original draft. F.C.: conceptualization, writing-Review and editing, resources. A.B.: formal analysis. V.B.: project administration, supervision, resources. S.C.: data curation, visualization. C.B.: conceptualization, validation. All authors have read and agreed to the published version of the manuscript.

Funding: A six-month scholarship was provided by the Pietro d'Abano Center for Thermal Studies to support research activity of junior researcher and co-author IA.

Conflicts of Interest: The authors declare there are no conflicts of interest.

\section{References}

1. Carretero, M.I. Clay minerals and their beneficial effects upon human health. A review. Appl. Clay Sci. 2002, 21, 155-163. [CrossRef]

2. Gomes, C.; Carretero, M.I.; Pozo, M.; Maraver, F.; Cantista, P.; Armijo, F.; Legido, J.L.; Teixeira, F.; Rautureau, M.; Delgado, R. Peloids and pelotherapy: Historical evolution, classification and glossary. Appl. Clay Sci. 2013, 75, 28-38. [CrossRef]

3. Quintela, A.; Terroso, D.; Ferreira da Silva, E.; Rocha, F. Certification and quality criteria of peloids used for therapeutic purposes. Clay Miner. 2012, 47, 441-451. [CrossRef]

4. Veniale, F.; Bettero, A.; Jobstraibizer, P.G.; Setti, M. Thermal muds: Perspectives of innovations. Appl. Clay Sci. 2007, 36, 141-147. [CrossRef]

5. Kulikov, A.G.; Turova, E.A.; Zaytseva, T.N. The role and significance of the physical factors in the treatment and prevention of erosive-ulcerative lesions of the stomach and duodenum. Vopr. Kurortol. Fizioter. Lech. Fiz. Kult. 2018, 95, 75-82. [CrossRef]

6. Antonelli, M.; Donelli, D.; Fioravanti, A. Effects of balneotherapy and spa therapy on quality of life of patients with knee osteoarthritis: A systematic review and meta-analysis. Rheumatol. Int. 2018, 38, 1807. [CrossRef]

7. Beasley, J.; Ward, L.; Knipper-Fisher, K.; Hughes, K.; Lunsford, D.; Leiras, C. Conservative therapeutic interventions for osteoarthritic finger joints: A systematic review. J. Hand. Ther. 2018, 32, 153-164. [CrossRef]

8. Fraioli, A.; Mennuni, G.; Fontana, M.; Nocchi, S.; Ceccarelli, F.; Perricone, C.; Serio, A. Efficacy of Spa Therapy, Mud-Pack Therapy, Balneotherapy, and Mud-Bath Therapy in the Management of Knee Osteoarthritis. A Systematic Review. Biomed. Res. Int. 2018, 2018, 1042576. [CrossRef]

9. Karagülle, M.E. Mud therapy in rheumatic diseases. J. Jpn. Soc. Balneol. Climatol. Phys. Med. 2014, 77, 503-504.

10. Maeda, T.; Kudo, Y.; Horiuchi, T.; Makino, N. Clinical and anti-aging effect of mud-bathing therapy for patients with fibromyalgia. Mol. Cell. Biochem. 2018, 444, 87. [CrossRef] [PubMed]

11. Weber-Rajek, M.; Lulinska-Kuklik, E.; Orlowska, K.; Czerniachowska, I.; Radziminska, A.; Moska, W. Evaluating the effectiveness of various forms of physical therapy in low back pain treatment. Trends Sport Sci. 2016, 23, 147-154.

12. Veniale, F.; Barberis, E.; Carcangiu, G.; Morandi, N.; Setti, M.; Tamanini, M.; Tessier, D. Formulation of muds for pelotherapy: Effects of maturation by different mineral waters. Appl. Clay Sci. 2004, 25, 135-148. [CrossRef]

13. Borda, L.J.; Perper, M.; Keri, J.E. Treatment of seborrheic dermatitis: A comprehensive review. J. Dermatolog. Treat. 2018, 30, 158-169. [CrossRef]

14. Musayev, A.V.; Imamverdiyeva, S.S.; Kerimbeyli, U.S. Pelotherapy of patients with diabetic poloyneuropathy: A clinical-immunological study. Zhurnal Nevrol. Psikhiatr. Im. S S Korsakova 2008, 108, 17-23. 
15. Masiero, S.; Litwocenko, S.; Agostini, F. Rehabilitation in an Italian thermal setting: A new therapeutic strategy for patients with musculoskeletal disability-The results of an Italian survey. Int. J. Biometeorol. 2020, 64, 951-954. [CrossRef] [PubMed]

16. Antonelli, M.; Donelli, D. Effects of balneotherapy and spa therapy on levels of cortisol as a stress biomarker: A systematic review. Int. J. Biometeorol. 2018, 62, 913. [CrossRef]

17. Tateo, F.; Agnini, C.; Carraro, A.; Giannossi, M.L.; Margiotta, S.; Medici, L.; Finizio, F.E. Short-term and long-term maturation of different clays for pelotherapy in an alkaline-sulphate mineral water (Rapolla, Italy). Appl. Clay Sci. 2010, 50, 503-511. [CrossRef]

18. Veneto Region. Regional Law 10/10/1989 n. 40 "Disciplina Della Ricerca, Coltivazione e Utilizzo Delle Acque Minerali e Termali"; Published on B.U.R. n. 58/1989; Regione del Veneto: Venezia, Italy, 1989.

19. Pozo, M.; Carretero, M.I.; Maraver, F.; Pozo, E.; Gomez, I.; Armijo, F.; Martin Rubi, J.A. Composition and physico-chemical properties of peloids used in Spanish spas: A comparative study. Appl. Clay Sci. 2013, 83, 270-279. [CrossRef]

20. Carretero, M.I.; Pozo, M.; Legido, J.L.; Fernandez-Gonzalez, M.V.; Delgado, R.; Gomez, L.; Armijo, F. Assessment of three Spanish clays for their use in pelotherapy. Appl. Clay Sci. 2014, 99, 131-143. [CrossRef]

21. Cantaluppi, C.; Fasson, A.; Ceccotto, F.; Cianchi, A.; Degetto, S. Radionuclides Concentration in Water and Mud of Euganean Thermal District. Int. J. Environ. Res. 2014, 8, 237-248.

22. De Zarate, J.M.; Hita, J.L.; Khayet, M.; Legido, J.L. Measurement of the thermal conductivity of clays used in pelotherapy by the multi-current hot-wire technique. Appl. Clay Sci. 2010, 50, 423-426. [CrossRef]

23. Tateo, F.; Ravaglioli, A.; Andreoli, C.; Bonina, F.; Coiro, V.; Degetto, S.; Giaretta, A. The in-vitro percutaneous migration of chemical elements from a thermal mud for healing use. Appl. Clay Sci. 2009, 44, 83-94. [CrossRef]

24. Carretero, M.I.; Pozo, M.; Martín-Rubí, J.A.; Pozo, E.; Maraver, F. Mobility of elements in interaction between artificial sweat and peloids used in Spanish spas. Appl. Clay Sci. 2010, 48, 506-515. [CrossRef]

25. Tolomio, C.; Ceschi-Berrini, C.; Moschin, E.; Galzigna, L.; Galzigna, L. Colonization by diatoms and antirheumatic activity of thermal mud. Cell Biochem. Funct. 1999, 17, 29-33. [CrossRef]

26. Poli, A.; Romano, I.; Cordella, P.; Orlando, P.; Nicolaus, B.; Berrini, C.C. Anoxybacillus thermarum sp. nov., a novel thermophilic bacterium isolated from thermal mud in Euganean hot springs, Abano Terme, Italy. Extremophiles 2009, 13, 867-874. [CrossRef] [PubMed]

27. Paduano, S.; Valeriani, F.; Romano-Spica, V.; Bargellini, A.; Borella, P.; Marchesi, I. Microbial biodiversity of thermal water and mud in an Italian spa by metagenomics: A pilot study. Water Sci. Technol. Water Supply 2018, 18, 1456-1465. [CrossRef]

28. Galzigna, L.; Lalli, A.; Moretto, C.; Bettero, A. Maturation of thermal mud controlled conditions and identification of an anti-inflammatory fraction. J. Phys. Rehabil. Med. 1995, 5, 196-199.

29. Scaglia, M.; Strosselli, M.; Grazioli, V.; Gatti, S.; Bernuzzi, A.M.; de Jonckheere, J.F. Isolation and identification of pathogenic Naegleria australiensis (Amoebida, Vahlkampfidae) from a spa in northern Italy. Appl. Environ. Microbiol. 1983, 46, 1282-1285. [CrossRef] [PubMed]

30. Sanchez-Espejo, R.; Aguzzi, C.; Cerezo, P.; Salcedo, I.; López-Galindo, A. Folk pharmaceutical formulations in western Mediterranean: Identification and safety of clays used in pelotherapy. J. Ethnopharmacol. 2014, 155, 810-814. [CrossRef]

31. Karakaya, M.; Karakaya, N.; Vural, H. Microbiological Viewpoint to Pelotherapy from Turkey. Ulutas Med. J. 2016, 2, 107. [CrossRef]

32. Quintela, A.; Terroso, D.; Almeida Salomè, F.P.; Reis, P.; Moura, A.; Correia, A.; da Silva, E.F.; Forjaz, V.; Rocha, F. Geochemical and microbiological characterization of some Azorean volcanic muds after maturation. Res. J. Chem. Environ. 2010, 14, 66-74.

33. Veneto Region. Disciplinare per la Tutela del Marchio Collettivo D'origine "Fango del Bacino Termale Euganeo"; Regione del Veneto: Venezia, Italy, 2013.

34. Byappanahalli, M.N.; Nevers, M.B.; Korajkic, A.; Staley, Z.R.; Harwood, V.J. Enterococci in the Environment. Microbiol. Mol. Biol. Rev. 2012, 76, 685-706. [CrossRef] [PubMed]

35. Stewart, S.E.; Parker, M.D.; Amézquita, A.; Pitt, T.L. Microbiological risk assessment for personal care products. Int. J. Cosmet. Sci. 2016, 38, 634-645. [CrossRef] [PubMed] 
36. Kim, H.W.; Seok, Y.S.; Cho, T.J.; Rhee, M.S. Risk factors influencing contamination of customized cosmetics made on-the-spot: Evidence from the national pilot project for public health (Scientific Reports, (2020), 10, 1, (1561), 10.1038/s41598-020-57978-9). Sci. Rep. 2020, 10, 1-9. [CrossRef]

37. Gomes, M.; Vieira, H.; Vale, F.P. Characterization, validation and application of a DNA microarray for the detection of mandatory and other waterborne pathogens. J. Biochem. 2015, 158, 393-401. [CrossRef]

(C) 2020 by the authors. Licensee MDPI, Basel, Switzerland. This article is an open access article distributed under the terms and conditions of the Creative Commons Attribution (CC BY) license (http://creativecommons.org/licenses/by/4.0/). 